\title{
A modified recursive partitioning analysis for predicting overall survival in patients with non-small cell lung cancer and central nervous system metastases
}

\author{
Thiago Pimentel Muniz, Victor Hugo Fonseca de Jesus, Victor Aurélio Ramos Sousa, Malu Viter da Rosa \\ Barbosa, Vladmir Cláudio Cordeiro de Lima
}

Department of Medical Oncology, A.C. Camargo Cancer Center, São Paulo, SP, Brazil

Contributions: Conception and Design: TP Muniz, VC de Lima; (II) Administrative Support: TP Muniz, VC de Lima, VH de Jesus; (III) Provision of study materials and patients: All authors; (IV) Collection and assembly of data: TP Muniz, VA Sousa, MR Barbosa; (V) Data analysis and interpretation: VH de Jesus, TP Muniz, VC de Lima; (VI) Manuscript writing: All authors; (VII) Final approval of manuscript: All authors.

Correspondence to: Thiago Pimentel Muniz. Department of Medical Oncology, A.C. Camargo Cancer Center, Rua Professor Antônio Prudente, 211, Liberdade, CEP: 01509-010, São Paulo, SP, Brazil. Email: thiagomuniz88@gmail.com.

\begin{abstract}
Background: Non-small cell lung cancer (NSCLC) is a major cause of brain metastases. Nonetheless, patients with central nervous system (CNS) spread are poorly represented in clinical trials. We sought to evaluate the overall survival (OS) of patients with NSCLC and CNS metastases.

Methods: Patients with NSCLC and CNS metastases treated at A. C. Camargo Cancer Center from January 2007 to December 2017 were selected. The primary endpoint was OS following the diagnosis of CNS metastasis. The Kaplan-Meier method was applied to calculate OS. Prognostic factors were assessed by the Cox Proportional Hazards model. As an exploratory analysis, a survival tree was generated based upon the two most statistically significant variables in the multivariate model and one additional clinically meaningful variable.

Results: In total, 311 patients were included. Median OS was 10.3 months (95\% CI, 8.7-13.1 months). ECOG performance status 2-4 (HR 2.12; 95\% CI, 1.40-3.20; $\mathrm{P}<0.01$ ) and the absence of a driver mutation (HR 3.30; 95\% CI, 1.85-5.90; $\mathrm{P}<0.01$ ) were strongly associated with worse OS. A Modified Recursive Partitioning Analysis (mRPA) was developed based on the curves generated by the survival tree. mRPA stratified our cohort in four subgroups with significantly different OS (3.1 to 43 months) and it outperformed both RPA and GPA in predicting OS in our population.

Conclusions: OS in our cohort was better than previously reported. However, prognosis is widely variable and is mostly dictated by performance status and the presence of a driver mutation.
\end{abstract}

Keywords: Non-small cell lung cancer (NSCLC); brain metastases, prognosis, overall survival (OS)

Submitted May 05, 2019. Accepted for publication Aug 12, 2019.

doi: $10.21037 /$ jtd.2019.08.115

View this article at: http://dx.doi.org/10.21037/jtd.2019.08.115

\section{Introduction}

Non-small cell lung cancer (NSCLC) is a heterogeneous group of diseases with several molecular subtypes (1). A common problem across all types of NSCLC is its proclivity to spread to the central nervous system (CNS). Thirty to fifty percent of patients diagnosed with stage IV disease have brain metastasis (2) and about fifty percent of patients with stage III disease treated with chemoradiation will develop brain lesions during follow-up (3). The frequency is even higher in patients with driver mutations: brain metastases can occur in up to $58.3 \%$ of patients with ALK rearrangements and $46.7 \%$ of patients with EGFR mutations at 3 years of treatment (4). In contrast to parenchymatous dissemination, leptomeningeal spread is an 
uncommon event and it occurs in about $5 \%$ of patients with NSCLC (5).

Albeit brain metastases from NSCLC are a frequent problem, patients with brain metastases are usually excluded from clinical trials. Studies evaluating the role of chemotherapy in this scenario are mostly retrospective or small prospective phase II trials with limited power (6-14). Most trials evaluating immunotherapy in the first- or second-line setting included few patients with brain lesions and the majority of them did not report the treatment efficacy in this subgroup (15-21), an exception being the KEYNOTE-189 trial, in which the combination of pembrolizumab, carboplatin and pemetrexed resulted in a $64 \%$ reduction of the risk of death in patients with brain metastases (22). Anti$A L K$ tyrosine kinase inhibitors (TKI) promote high rates of intracranial response and prolonged intracranial progression free survival and intracranial duration of response, but overall survival (OS) data for patients with brain metastases are largely underreported (23-27). In small prospective studies, first-generation anti-EGFR TKIs have been associated with intracranial response rates around 90\% (28) and median OS reaching 22 months (29); nonetheless, there is no OS data for patients with brain metastasis treated with the third-generation TKI osimertinib (30,31).

The median OS following the diagnosis of brain metastasis from NSCLC is only 7 months. However, prognosis is heterogeneous and depends on the clinical presentation at the time of diagnosis (32). Since the 1990's, prognostic tools have been developed to help to select the best approach for these patients, such as the Recursive Partitioning Analysis (RPA). It divides patients in three classes, based on the patients' Karnofsky Performance Status (KPS), age, the primary tumor control status, and the presence or absence of extracranial metastases, with median survival ranging from 2.3 to 7.1 months (33). In 2012, another risk assessment tool, the NSCLC Graded Prognostic Assessment (GPA), was published. This tool attributes numeric scores to the following prognostic factors: KPS, age, extracranial metastasis, and number of brain lesions. The sum of the scores can vary from 0 to 4: patients with total scores 0 to 1 have an estimated OS of 3 months while patients with scores 3.5 to 4 achieve 14.8 months of OS (32). However, none of them take into account the current molecular classification of NSCLC.

The aim of this study was to evaluate the OS of NSCLC patients with disease spread to the CNS and to perform an analysis of the prognostic factors associated with this outcome. We also sought to devise a prognostic model for patients with NSCLC with brain metastases.

\section{Methods}

\section{Study design, patient selection and data collection}

This is a descriptive and analytical, retrospective, single center study. We included patients 18 years of age or older with histologically or cytologically proven NSCLC and who presented metastatic disease spread to the CNS (parenchyma and/or leptomeninges, diagnosed either by imaging studies or histological/cytological analysis), treated at A. C. Camargo Cancer Center from January 2007 to December 2017 (see Supplementary Appendix for detailed inclusion and exclusion criteria). Patients who received only best supportive care were also included. Patients with small cell lung cancer, large cell neuroendocrine tumor of the lung, a second malignant neoplasia diagnosed concomitantly or up to five years before the diagnosis of NSCLC, those who did not start treatment at A. C. Camargo Cancer Center, those who were followed at another institution or those whose available clinical information in the medical records was considered insufficient were excluded.

We searched the A. C. Camargo Cancer Center electronic medical records for the International Classification of Diseases $10^{\text {th }}$ revision (ICD-10) C34 to identify patients diagnosed with lung cancer. Demographics and clinical information were collected from the patients' medical charts.

This study was approved by the Institutional Review Board (IRB) of A. C. Camargo Cancer Center (number 3.106.042), in accordance with the principles laid down in the Declaration of Helsinki. Due to its retrospective nature, the results of this study will not affect the treatment of the patients enrolled. Because data were to be collected from patients' medical records, the IRB considered that an Informed Consent Form was unnecessary. However, the authors declare that raw data were shared amongst the authors only, and the data presented herein do not identify any patient, since they are presented all together, securing their privacy and anonymity.

\section{Endpoints definition}

The primary endpoint was OS, which was defined as the time in months between the date of diagnosis of the CNS 
metastasis and death by any cause. Secondary endpoint was intracranial progression free-survival, defined as the time in months between the date of diagnosis of CNS metastasis and any evidence of intracranial disease progression (increase in size of a previous lesion, the appearance of new lesions or any evidence of leptomeningeal spread de novo or recurrent) or death by any cause. Intracranial progression was defined by the attending physician at time the patients were evaluated.

\section{Statistical analyses}

Numeric variables were described by their median values and interquartile ranges (IQR); categorical variables were described by their absolute and relative frequencies. Median OS and intracranial progression-free survival were calculated by the Kaplan-Meier method. The logrank test was used for comparisons between subgroups. We used the reverse Kaplan-Meier method to calculate the median follow-up. Patients were censored at the time when they were last known to be alive. To assess the potential prognostic role of clinical or pathologic features on OS and intracranial progression-free survival, we used the Cox Proportional Hazards model. Variables with $\mathrm{P}<0.20$ at univariate analysis were used to generate a multivariate model. Eastern Cooperative Oncology Group performance status score (ECOG PS), the presence of driver mutations (EGFR, ALK or ROS1) and the number of brain metastases were evaluated as forced entrance variables in the multivariate model. For both univariate and multivariate analyses, only patients with exclusive parenchymatous metastases (i.e., patients without meningeal spread) and complete data were included. We used backward variable elimination in accordance to likelihood ratio tests and Akaike Information Criteria (AICs) to select the most adequate multivariate model.

As an exploratory analysis, the two most significant variables in the multivariate model were selected to generate a survival tree for the outcome OS. It has been defined that a third variable would be included, chosen according to the visual inspection of the curves of the generated groups. In order to generate the survival tree, we used the likelihood method, as described by LeBlanc and Crowley, without tree pruning. We compared the performance of the survival model generated by the survival tree analysis in our population (Modified Recursive Partitioning Analysis) with that of RPA and GPA using C-statistic and AIC. Also, we used non-parametric Bootstrap with resampling (200 repetitions) to perform internal validation of our findings and further compare the performance of the three different prognostic models. A two-sided $\mathrm{P}<0.05$ was considered statistically significant. All statistical analyses were made in the R software, version 3.4.0.

\section{Results}

We initially identified 2,725 patients with the diagnosis of lung cancer. One thousand and seven hundred and ninetytwo patients did not meet the inclusion criteria and were excluded. Nine-hundred and thirty-three patients with NSCLC were included in the initial databank; however, 633 patients never presented CNS metastasis. Therefore, 311 patients with CNS metastases were included in this analysis (Figure $S 1$ in the Supplementary Appendix). Clinical characteristics of the patients are shown in Table 1.

Median age was 60 years (IQR, 54-68 years) and 50.8\% were female. Nearly one third of the population was never smoker. Two hundred and twenty-six (72.7\%) patients had an ECOG PS 0 or 1 and adenocarcinoma constituted the most frequent histology (82.0\%). Patients mostly presented exclusive parenchymatous disease $(92.3 \%)$. A known driver mutation (EGFR, ALK or ROS1) was detected in $18.3 \%$ of the patients.

Local therapy (radiotherapy, neurosurgery or intrathecal chemotherapy) was most frequently chosen as the primary approach to the CNS metastases and it was usually used alone rather than concurrently with systemic modalities. Whole brain radiotherapy (WBRT) was the most common approach used (49.8\%). When concurrent therapy was chosen as the primary approach, chemotherapy was the systemic modality most commonly given in combination with some sort of local treatment (9.3\%). Table 2 shows the different modalities of therapy patients received after the diagnosis of CNS metastasis.

The median intracranial progression-free survival was 7.1 months [95\% confidence interval (CI), 6.1-8.6] and median OS was 10.3 months (95\% CI, 8.7-13.1 months) (Figure 1A,B). We compared the intracranial progression-free survival and OS between patients with exclusively parenchymatous lesions and those with leptomeningeal disease (with or without brain parenchymatous lesions). We found a statistically significant difference in both outcomes between these subgroups: intracranial progression-free survival was 2.5 months (95\% CI, 1.4-6.4 months) for patients with leptomeningeal disease and 7.6 months (95\% CI, 6.4-8.9 months) for patients with exclusively parenchymatous 
Table 1 Clinical characteristics of enrolled patients

\begin{tabular}{|c|c|}
\hline Variable & Value \\
\hline Age (years) & 60 [54-68] \\
\hline \multicolumn{2}{|l|}{ Sex } \\
\hline Male & $153(49.2)$ \\
\hline Female & $158(50.8)$ \\
\hline \multicolumn{2}{|l|}{ Smoking status ${ }^{a}$} \\
\hline Active smoker & $76(24.4)$ \\
\hline Never smoker & $100(32.2)$ \\
\hline Former smoker & $124(39.9)$ \\
\hline Unavailable & $11(3.5)$ \\
\hline Smoking load (pack-years) & $32[10-50]$ \\
\hline Largest diameter of the largest lesion $(\mathrm{mm})$ & 15 [9-23] \\
\hline Charlson Comorbidity Index & $8[7-9]$ \\
\hline \multicolumn{2}{|l|}{ ECOG performance status } \\
\hline $0-1$ & $226(72.7)$ \\
\hline $2-4$ & $48(15.4)$ \\
\hline Unavailable & $37(11.9)$ \\
\hline \multicolumn{2}{|l|}{ Histology } \\
\hline Adenocarcinoma & $255(82.0)$ \\
\hline Non-Adenocarcinoma & $42(13.5)$ \\
\hline Non evaluable & $14(4.5)$ \\
\hline \multicolumn{2}{|l|}{ RPA } \\
\hline I & $18(5.8)$ \\
\hline II & $271(87.1)$ \\
\hline III & $22(7.1)$ \\
\hline GPA & $1.5[1-2]$ \\
\hline \multicolumn{2}{|l|}{ Site of metastasis } \\
\hline Parenchyma & $287(92.3)$ \\
\hline Meninges & $18(5.8)$ \\
\hline Parenchyma and Meninges & $6(1.9)$ \\
\hline \multicolumn{2}{|l|}{ Time of diagnosis $^{a}$} \\
\hline Synchronous & $239(76.8)$ \\
\hline Metachronous & 72 (23.2) \\
\hline \multicolumn{2}{|l|}{ Known driver mutation $^{\mathrm{b}}$} \\
\hline Yes & 57 (18.3) \\
\hline No & $254(81.7)$ \\
\hline
\end{tabular}

Table 1 (continued)
Table 1 (continued)

\begin{tabular}{lc}
\hline Variable & Value \\
\hline Number of extracranial sites ${ }^{a}$ & $56(18.0)$ \\
0 & $91(29.3)$ \\
2 & $82(26.4)$ \\
3 & $48(15.4)$ \\
4 or more & $34(10.9)$ \\
Number of lesions in CNS & \\
$0^{c}$ & $18(5.8)$ \\
1 & $95(30.5)$ \\
2 & $38(12.2)$ \\
3 & $24(7.7)$ \\
4 or more & $136(43.7)$ \\
\hline
\end{tabular}

Data are shown as median [IQR] or frequency (\%). a, see supplementary material for definitions of categories; b, EGFR, $A L K$ or ROS1; c, patients with exclusive meningeal disease. IQR, interquartile range; ECOG, Eastern Cooperative Oncology Group; RPA, recursive partitioning analysis; GPA, graded prognostic assessment; CNS, central nervous system.

lesions ( $\mathrm{P}<0.01$ ); OS was 2.5 months (95\% CI, 1.4-10.3 months) for patients with leptomeningeal spread compared to 11 months (95\% CI, 8.8-13.4 months) for patients with brain lesions without leptomeningeal spread $(\mathrm{P}<0.01$; Figures $S 2, S 3$, respectively, found in Supplementary Appendix).

For OS, the adverse prognostic factors in univariate analysis were: age, Charlson comorbidity index, the largest diameter of the largest lesion, ECOG PS 2-4, the absence of a known driver mutation, and the number of extracranial metastatic sites. In multivariate analysis, Charlson comorbidity index, the absence of known driver mutation, ECOG PS 2-4, and the number of extracranial metastatic sites remained as independent prognostic factors, while metachronous brain metastasis was associated with improved OS. Univariate and multivariate analyses for OS are shown in Table 3.

The prognostic factors statistically associated with intracranial progression or death in univariate analysis were: age, the largest diameter of the largest lesion, ECOG-PS $2-4$, the absence of a known driver mutation, the number of brain lesions, the number of extracranial metastatic sites, and the time of the diagnosis of the CNS metastasis 
Table 2 Modalities of treatment applied after CNS metastasis diagnosis

\begin{tabular}{|c|c|}
\hline Variable & Value \\
\hline \multicolumn{2}{|l|}{ Type of treatment } \\
\hline Local & $199(40.0)$ \\
\hline Systemic & $17(5.5)$ \\
\hline Local and Systemic & $79(25.4)$ \\
\hline Not treated & $16(5.1)$ \\
\hline \multicolumn{2}{|l|}{ Systemic treatment only ${ }^{a}$} \\
\hline Chemotherapy & $10(3.2)$ \\
\hline Tyrosine Kinase Inhibitor & $7(2.3)$ \\
\hline Immunotherapy & $0(0)$ \\
\hline Not applicable & $278(89.4)$ \\
\hline Not treated & $16(5.1)$ \\
\hline \multicolumn{2}{|l|}{ Concurrent systemic and local treatment ${ }^{a}$} \\
\hline Chemotherapy & $29(9.3)$ \\
\hline Tyrosine Kinase Inhibitor & $19(6.1)$ \\
\hline Immunotherapy & $4(1.3)$ \\
\hline Not applicable & $226(72.7)$ \\
\hline Not treated & $33(10.6)$ \\
\hline \multicolumn{2}{|l|}{ Upfront systemic treatment ${ }^{a}$} \\
\hline Chemotherapy & $22(7.1)$ \\
\hline Tyrosine kinase inhibitor & $4(1.3)$ \\
\hline Immunotherapy & $1(0.3)$ \\
\hline Not applicable & $251(80.7)$ \\
\hline Not treated & $33(10.6)$ \\
\hline \multicolumn{2}{|l|}{ Type of local treatment } \\
\hline WBRT & $155(49.8)$ \\
\hline Radiosurgery & $72(23.2)$ \\
\hline WBRT + radiosurgery & $3(1.0)$ \\
\hline Neurosurgery & $13(4.2)$ \\
\hline Neurosurgery + radiotherapy & $26(8.4)$ \\
\hline Intrathecal chemotherapy & $7(2.3)$ \\
\hline Intrathecal chemotherapy + radiotherapy & $2(0.6)$ \\
\hline Not treated & $33(10.6)$ \\
\hline
\end{tabular}

Data are shown as number (\%). a , see supplementary appendix for definition of categories. CNS, central nervous system; WBRT, whole brain radiotherapy. in relation to the primary tumor diagnosis. The diagnosis of a metachronous brain metastasis, in opposition to a synchronous brain metastasis, was associated with a reduced risk of intracranial progression or death. In multivariate analysis, ECOG PS 2-4, the absence of a known driver mutation and the number of brain metastasis remained as independent predictors of poor intracranial disease-free survival, while the diagnosis of a metachronous metastasis to the CNS remained as a harbinger of improved intracranial progression-free survival. Univariate and multivariate analyses for intracranial progression-free survival are presented in Table $S 1$ in Supplementary Appendix.

The two variables that most strongly contributed as prognostic factors for OS were ECOG PS and the presence of a known driver mutation; hence the survival tree was generated based upon these variables. The third most suitable variable was the number of brain lesions (Figure 2). Based on these three variables, we could distribute patients in our cohort in four prognostic classes, according to a Modified Recursive Partitioning Analysis (mRPA) (Table S2 in Supplementary Appendix). Patients in mRPA class I (ECOG $0-1$ and presence of a known driver mutation) had a median OS of 43 months; patients in mRPA class II (ECOG $0-1$ and no driver mutation, and single brain metastasis) had a median OS of 18 months; median OS for patients in mRPA class III (ECOG 0-1 and no driver mutation, and $>1$ brain metastasis) was 9.3 months; patients in mRPA class IV (ECOG 2-4) had only 3.1 months of OS. Survival curves according to mRPA are shown in Figure 3.

OS was not different for patients classified as RPA class I and II in our cohort (16.4 vs. 13.0 months; HR 1.3; 95\% CI, 0.57-2.96; P=0.53); however, OS for patients classified as RPA class III was significantly inferior (1.3 months; HR 6.46; 95\% CI, 2.42-17.26; $\mathrm{P}<0.01$ ) (Table $S 3$ in Supplementary Appendix). When we classified our cohort according to GPA, we found that patients with GPA score 1.5-2.5 had a statistically superior OS compared to patients with GPA score $0-1$ (13.3 vs. 7.1 months; HR 0.69; 95\% CI, 0.5-0.97; $\mathrm{P}=0.03)$. Patients with GPA score 3 achieved 29.5 months of OS (95\% CI, 7.9-NR); however, this was not statistically significant when compared to patients with GPA score 0-1 (HR 0.36; 95\% CI, 0.13-1.01; $\mathrm{P}=0.053)$. Besides, patients with GPA score $>3$ had a numerically inferior OS when compared to patients with GPA score 3 (19.5 vs. 29.5 months) (Table S4 in Supplementary Appendix). It should be noticed that there 

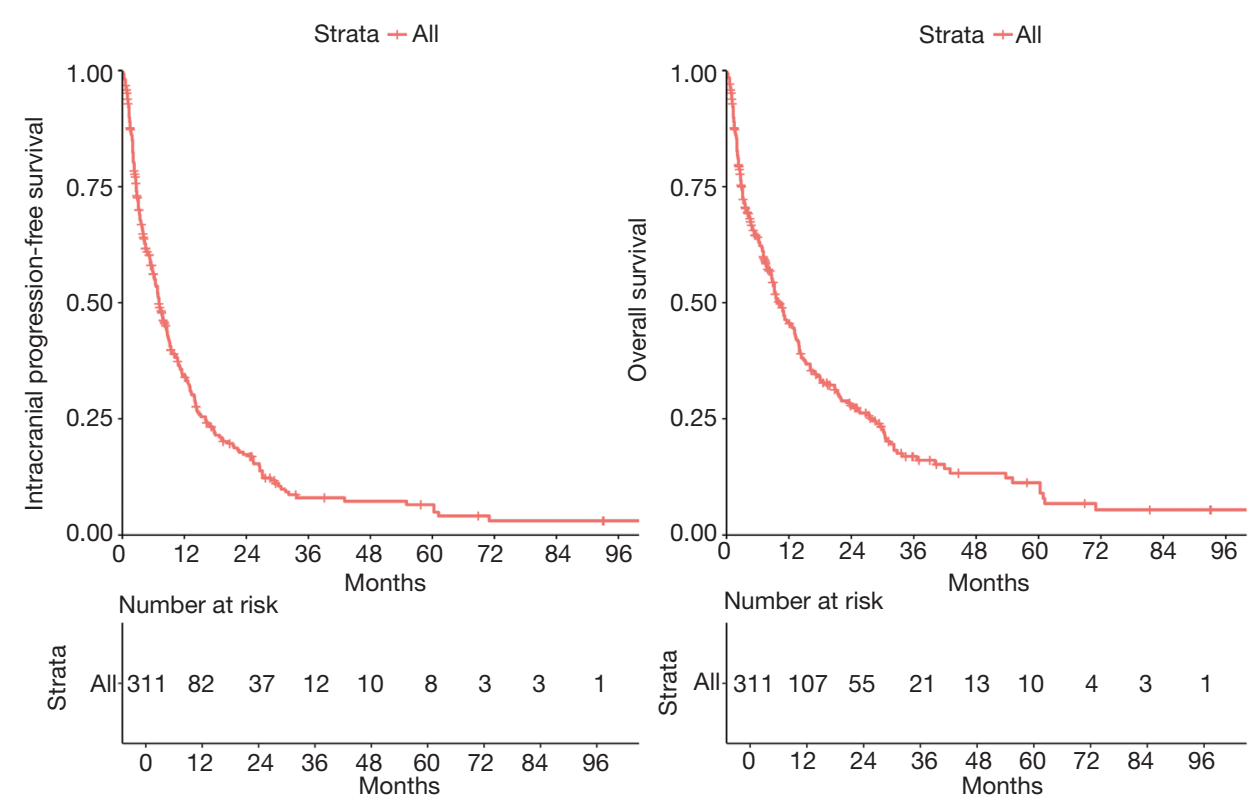

Figure 1 Kaplan-Meier curves of intracranial progression-free survival (A) and overall survival (B) in the studied population.

were only 16 patients with GPA score 3 or $>3$ (8 patients in each subgroup).

The prognostic stratification in our population was better described with mRPA than with RPA or GPA. The mRPA presented an increased concordance (C-statistic $=0.65)$ when compared to RPA $(\mathrm{C}$-statistic $=0.56)$ and GPA $(\mathrm{C}$-statistic $=0.59)$. Also, the AIC analysis demonstrated better performance of the mRPA (AIC $=1,328.4$ ) when compared to RPA (AIC $=1,344.1)$ and GPA (AIC $=1,356.1$ ). These findings held true after nonparametric Bootstrap (Table 55 in Supplementary Appendix).

\section{Discussion}

CNS metastases from NSCLC were a common event in our cohort. Thirty-three percent of NSCLC patients presented CNS spread, either at diagnosis or later in the course of the disease. This frequency is similar to what has been reported in other studies $(2,34)$. The higher frequency of patients with adenocarcinoma histology possibly reflects the increase in incidence and prevalence of this subtype in last decades, in contrast with the drop of squamous cell carcinoma and other NSCLC histologies (35). On the other hand, it can also indicate that lung adenocarcinomas have a greater tropism for the CNS than other histologies. The median age at diagnosis of CNS spread in our cohort was similar to other populations and it was lower than the median age of patients diagnosed with NSCLC without brain metastases (2).
Median OS in our cohort was longer than expected. In an analysis of patients with brain metastases from NSCLC treated with chemotherapy across Europe, OS was 7.2 months and OS among all patients (with and without CNS metastases) was 10.3 months (2). A retrospective Canadian series has also found an OS of 7.8 months (36). The OS in our cohort was comparable only to a German retrospective study in which all patients had their brain metastases and primary tumors resected (11.2 months) (37), and to metastatic NSCLC patients treated with chemotherapy and bevacizumab (9.3 months) or TKIs (10.3 months) (38). It should be pointed out that the minority of our patients had their brain lesions resected $(4.1 \%)$ or received TKIs as the initial treatment to their CNS metastases (2.2\%). The improvements in OS observed in this study might reflect the changes in staging procedures and the availability of better antineoplastic and supportive treatments during the study period.

Some of the adverse prognostic factors found in the multivariate analyses, such as the presence and the number of extracranial metastases and the performance status, were also described in other series $(39,40)$. The association of metachronous brain metastases with better OS was also reported by Enders et al. (37), although, in their study, this association was not statistically significant. The development of a metachronous brain metastasis might reflect a disease with less aggressive biological behavior or more sensitive to systemic treatment, which translates into 
Table 3 Univariate and multivariate analyses for overall survival ${ }^{2}$

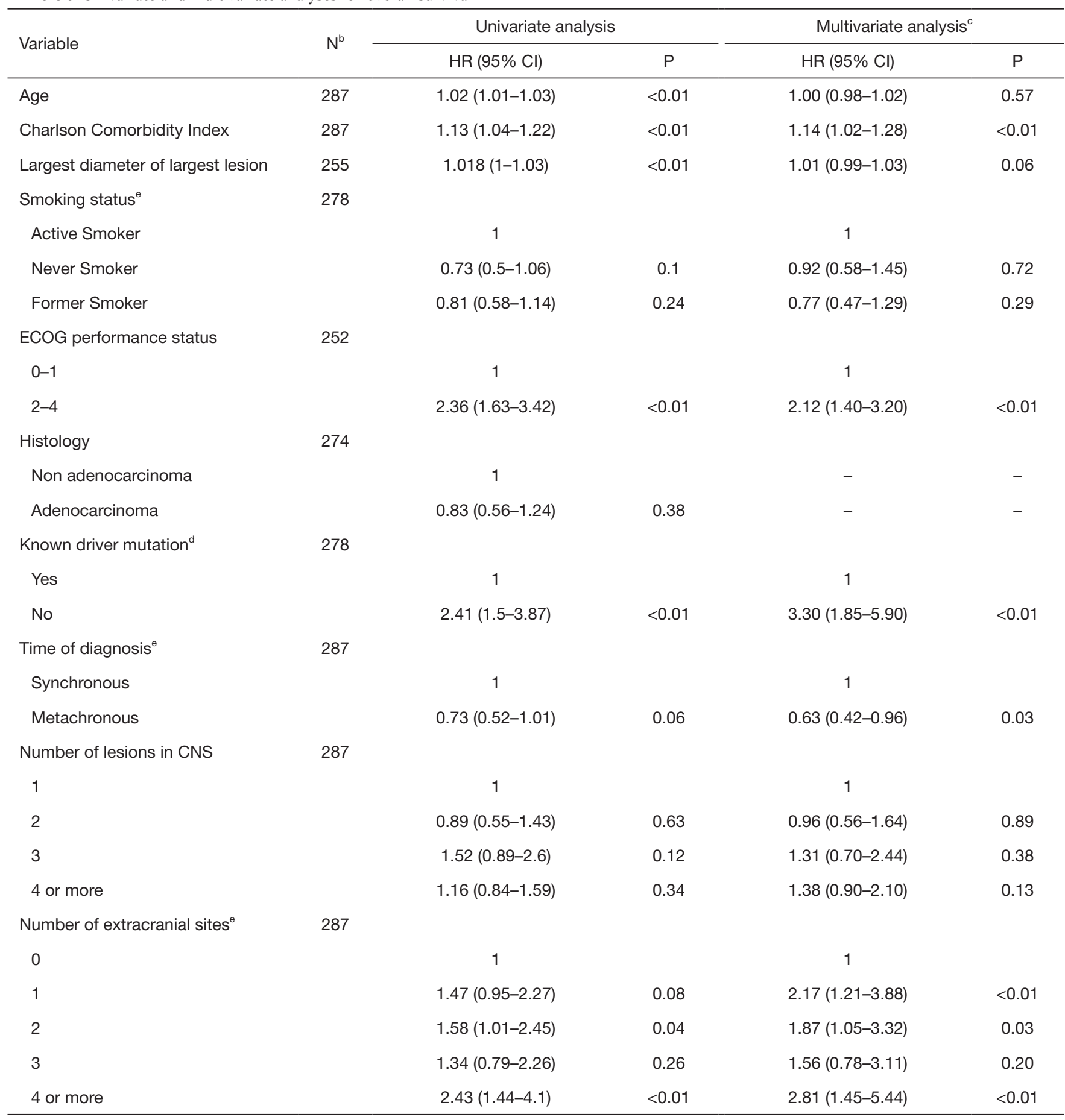

${ }^{a}$, patients with meningeal carcinomatosis, and without complete data were not included; ${ }^{b}$, number of patients with complete data for each variable in univariate analysis; ' , two hundred and eighteen patients were included for multivariate analysis; ${ }^{\mathrm{d}}$, EGFR, ALK or ROS1; e, see supplementary appendix for definition of categories. HR, hazard ratio; Cl, confidence interval; ECOG, Eastern Cooperative Oncology Group; CNS, central nervous system. 


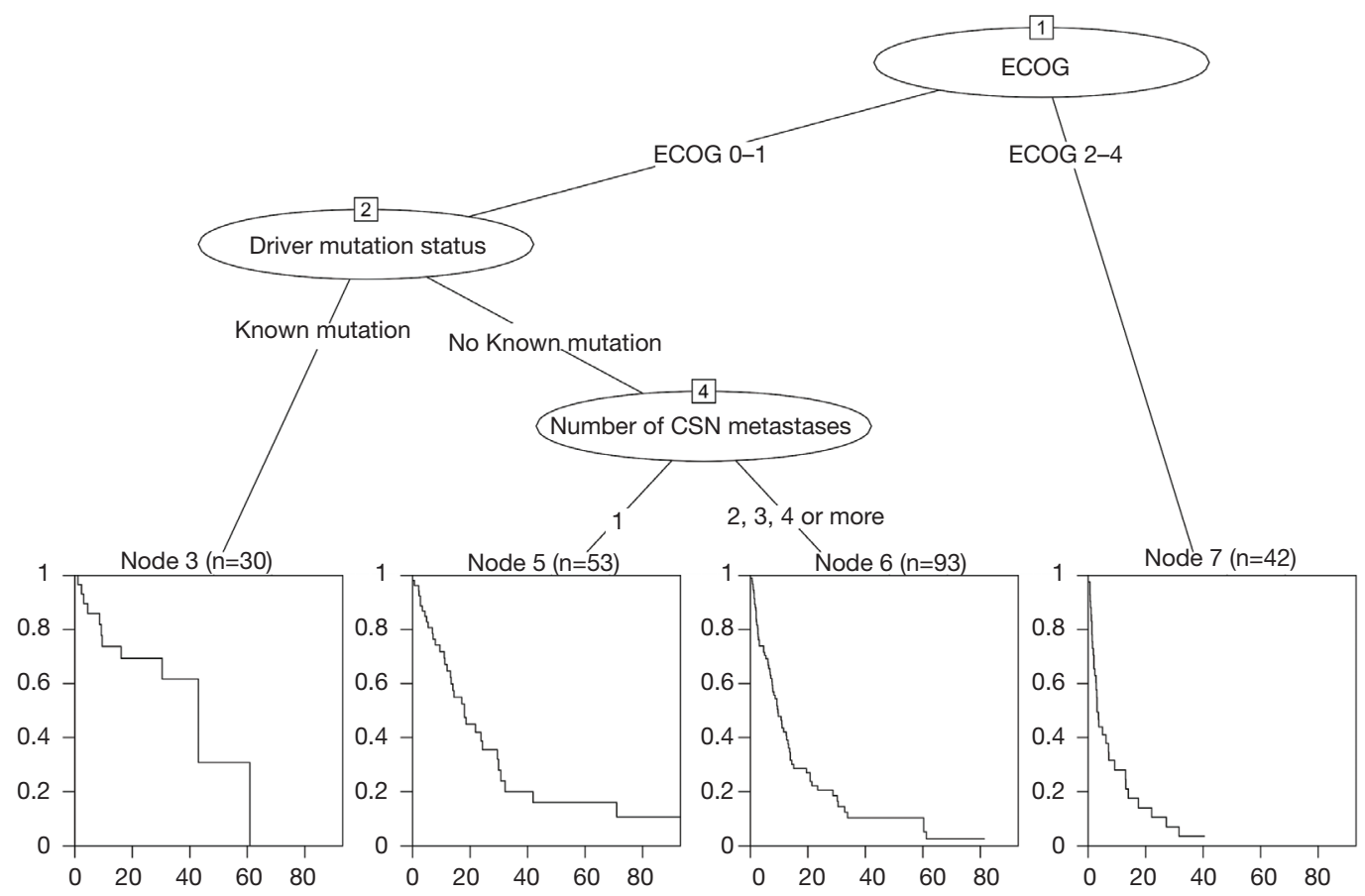

Figure 2 Survival tree for overall survival in patients with exclusive brain parenchymatous disease. ECOG, Eastern Cooperative Oncology Group; CNS, central nervous system.

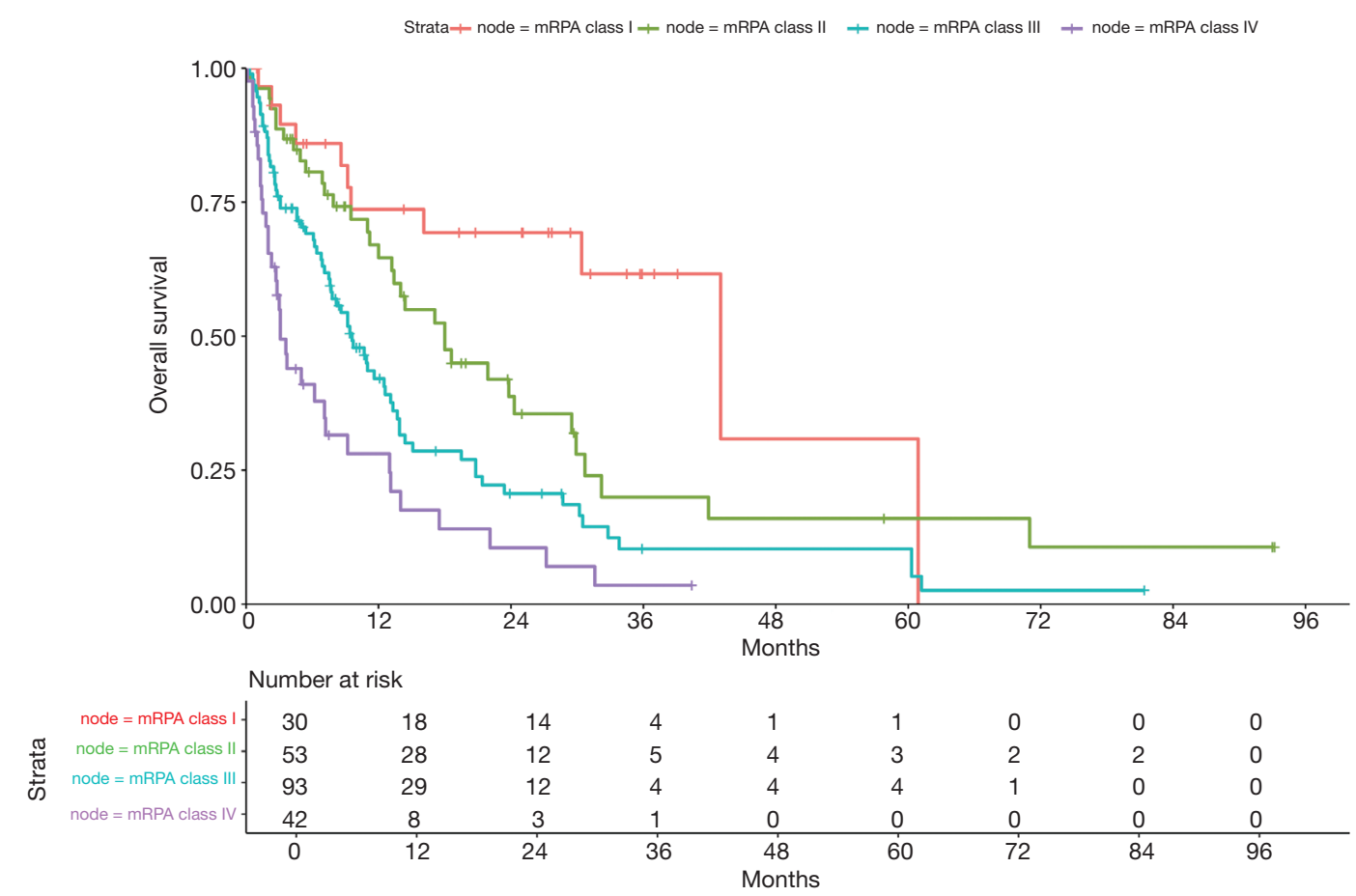

Figure 3 Overall survival curves according to modified recursive partitioning analysis (mRPA) classes in patients with exclusive parenchymatous disease. 
a longer control of extracranial metastases. Since late 90's, the control of extracranial disease has been recognized as an important prognostic factor for OS in patients with brain metastases from NSCLC (33).

In our cohort, RPA was ineffective in discriminating prognosis among patients classified as class I and class II. Additionally, patients in these classes had an OS longer than expected. It should be noted that the population upon which RPA was developed did not include patients solely with lung cancer (nearly $30 \%$ had other primary tumors, mainly breast cancer) (33), whilst our cohort included exclusively NSCLC patients. The factors associated with survival for other malignancies metastatic to the brain differ from those of lung cancer (32), what can explain, at least partially, the differences between the expected and observed survival time.

However, even when using NSCLC GPA (32), OS in our cohort did not conform to what was expected and patients with GPA score 3 had longer OS than patients with GPA score $>3$. Although specific for NSCLC, the database for GPA included patients treated from 1985 to 2007, while our database included patients treated between 2007 and 2017. The knowledge about the molecular biology of NSCLC has experienced a remarkable evolution during the recent years and, consequently, new treatments became available, leading to an improvement in prognosis. Therefore, GPA may no longer reflect the prognosis of patients with NSCLC and brain metastases.

Noteworthy, GPA has been recently updated and now includes the presence or absence of driver mutations (EGFR mutations or $A L K$ rearrangements) as prognostic factors (the LungMol-GPA) (41). Patients with 3.5-4 points in the LungMol-GPA (i.e., patients with a good performance status and a driver mutation) achieve 46 months of median OS, which is similar to the OS observed for patients in mRPA class I in our cohort (43 months). Another similarity between LungMol-GPA and mRPA is that when a patient has a good performance status and a driver mutation, the number of brain metastases loses relevance as a prognostic factor.

Regardless of the presence of a driver mutation, poor performance status is strongly associated with a shorter OS, usually no longer than 3 months, which can be observed in RPA (33), GPA (32), LungMol-GPA (41) and in our model as well. This reinforces the recommendation to treat such patients with best supportive care exclusively, since active treatment is unlikely to add any benefit in terms of OS. For instance, it has been demonstrated that best supportive care alone was non-inferior to radiation therapy plus best supportive care in patients with poor performance status; moreover, brain irradiation was associated with more toxic effects (3).

Patients with meningeal disease had only 2.5 months of median OS, similar to the results of a Canadian retrospective series, in which patients had a median OS of 3 months (42). In this analysis, WBRT had no impact on OS, but patients who received intrathecal chemotherapy $(n=6)$ achieved 18 months of OS. It is not possible to draw definitive conclusions based on these data, given the small number of patients and its retrospective nature; however, it is clear that, despite improvements in diagnosis and therapeutics of NSCLC, meningeal carcinomatosis still carries a dismal prognosis (43).

This study has some limitations. It is a retrospective analysis with possible biases inherent to this type of study. Patients were treated in a single reference cancer center and the data presented herein might not reflect the reality of other centers in Brazil. Furthermore, we could not perform a central review of the images and there is some degree of overfitting in the proposed prognostic model. However, this work includes a considerably large number of patients, which gives robustness to the data. Additionally, these patients represent real-life data gathered during 10 years from a national reference cancer center in Brazil. At last, we propose a new prognostic model that can assist physicians in deciding the best treatment approach for patients with brain metastases from NSCLC. Nevertheless, we acknowledge this model is exploratory and needs further external validation.

In conclusion, intracranial progression-free survival and OS in our cohort were better than previously reported in literature, possibly reflecting recent improvements in NSCLC treatment. ECOG performance status and the presence of a driver mutation have a major impact on OS of NSCLC patients with brain metastases. The Modified Recursive Partitioning Analysis outperformed RPA and GPA predicting OS in our cohort.

\section{Acknowledgments}

None.

\section{Footnote}

Conflict of Interest: The authors have no conflicts of interest to declare. 
Ethical Statement: The authors are accountable for all aspects of the work in ensuring that questions related to the accuracy or integrity of any part of the work are appropriately investigated and resolved. This study was approved by the Institutional Review Board (IRB) of A. C. Camargo Cancer Center (number 3.106.042), in accordance with the principles laid down in the Declaration of Helsinki. Due to its retrospective nature, the results of this study will not affect the treatment of the patients enrolled. Because data were to be collected from patients' medical records, the IRB considered that an Informed Consent Form was unnecessary.

\section{References}

1. Chan BA, Hughes BGM. Targeted therapy for non-small cell lung cancer: current standards and the promise of the future. Transl Lung Cancer Res 2015;4:36-54.

2. Moro-Sibilot D, Smit E, de Castro Carpeño J, et al. Nonsmall cell lung cancer patients with brain metastases treated with first-line platinum-doublet chemotherapy: Analysis from the European FRAME study. Lung Cancer 2015;90:427-32.

3. Mulvenna P, Nankivell M, Barton R, et al. Dexamethasone and supportive care with or without whole brain radiotherapy in treating patients with nonsmall cell lung cancer with brain metastases unsuitable for resection or stereotactic radiotherapy (QUARTZ): results from a phase 3 , non-inferiority, randomised trial. Lancet 2016;388:2004-14.

4. Rangachari D, Yamaguchi N, VanderLaan PA, et al. Brain metastases in patients with EGFR-mutated or ALKrearranged non-small-cell lung cancers. Lung Cancer 2015;88:108-11.

5. Cheng H, Perez-Soler R. Leptomeningeal metastases in non-small-cell lung cancer. Lancet Oncol 2018;19:e43-55.

6. Cortes J, Rodriguez J, Aramendia JM, et al. Frontline paclitaxel/cisplatin-based chemotherapy in brain metastases from non-small-cell lung cancer. Oncology 2003;64:28-35.

7. Fujita A, Fukuoka S, Takabatake H, et al. Combination chemotherapy of cisplatin, ifosfamide, and irinotecan with rhG-CSF support in patients with brain metastases from non-small cell lung cancer. Oncology 2000;59:291-5.

8. Cotto C, Berille J, Souquet PJ, et al. A phase II trial of fotemustine and cisplatin in central nervous system metastases from non-small cell lung cancer. Eur J Cancer 1996;32A:69-71.
9. Barlesi F, Gervais R, Lena H, et al. Pemetrexed and cisplatin as first-line chemotherapy for advanced nonsmall-cell lung cancer (NSCLC) with asymptomatic inoperable brain metastases: a multicenter phase II trial (GFPC 07-01). Ann Oncol 2011;22:2466-70.

10. Bailon O, Chouahnia K, Augier A, et al. Upfront association of carboplatin plus pemetrexed in patients with brain metastases of lung adenocarcinoma. Neuro Oncol 2012;14:491-5.

11. Bearz A, Garassino I, Tiseo M, et al. Activity of Pemetrexed on brain metastases from Non-Small Cell Lung Cancer. Lung Cancer 2010;68:264-8.

12. Cagney DN, Martin AM, Catalano PJ, et al. Impact of pemetrexed on intracranial disease control and radiation necrosis in patients with brain metastases from non-small cell lung cancer receiving stereotactic radiation. Radiother Oncol 2018;126:511-8.

13. Quadvlieg V, Bosquee L, Gustin M, et al. Frontline gemcitabine and cisplatin based chemotherapy in patients with NSCLC inoperable brain metastases. J Clin Orthod 2004;22:7117.

14. Besse B, Le Moulec S, Mazières J, et al. Bevacizumab in Patients with Nonsquamous Non-Small Cell Lung Cancer and Asymptomatic, Untreated Brain Metastases (BRAIN): A Nonrandomized, Phase II Study. Clin Cancer Res 2015;21:1896-903.

15. Brahmer J, Reckamp KL, Baas P, et al. Nivolumab versus Docetaxel in Advanced Squamous-Cell Non-Small-Cell Lung Cancer. N Engl J Med 2015;373:123-35.

16. Borghaei H, Paz-Ares L, Horn L, et al. Nivolumab versus Docetaxel in Advanced Nonsquamous Non-Small-Cell Lung Cancer. N Engl J Med 2015;373:1627-39.

17. Rittmeyer A, Barlesi F, Waterkamp D, et al. Atezolizumab versus docetaxel in patients with previously treated non-small-cell lung cancer (OAK): a phase 3, openlabel, multicentre randomised controlled trial. Lancet 2017;389:255-65.

18. Reck M, Rodríguez-Abreu D, Robinson AG, et al. Pembrolizumab versus Chemotherapy for PD-L1Positive Non-Small-Cell Lung Cancer. N Engl J Med 2016;375:1823-33.

19. Paz-Ares L, Luft A, Vicente D, et al. Pembrolizumab plus Chemotherapy for Squamous Non-Small-Cell Lung Cancer. N Engl J Med 2018;379:2040-51.

20. Hellmann MD, Ciuleanu T-E, Pluzanski A, et al. Nivolumab plus Ipilimumab in Lung Cancer with a High Tumor Mutational Burden. N Engl J Med 2018;378:2093-104. 
21. Socinski MA, Jotte RM, Cappuzzo F, et al. Atezolizumab for First-Line Treatment of Metastatic Nonsquamous NSCLC. N Engl J Med 2018;378:2288-301.

22. Gandhi L, Rodríguez-Abreu D, Gadgeel S, et al. Pembrolizumab plus Chemotherapy in Metastatic NonSmall-Cell Lung Cancer. N Engl J Med 2018;378:2078-92.

23. Solomon BJ, Mok T, Kim DW, et al. First-line crizotinib versus chemotherapy in ALK-positive lung cancer. N Engl J Med 2014;371:2167-77.

24. Hida T, Nokihara H, Kondo M, et al. Alectinib versus crizotinib in patients with ALK-positive non-small-cell lung cancer (J-ALEX): an open-label, randomised phase 3 trial. Lancet 2017;390:29-39.

25. Peters S, Camidge DR, Shaw AT, et al. Alectinib versus Crizotinib in Untreated ALK-Positive Non-Small-Cell Lung Cancer. N Engl J Med 2017;377:829-38.

26. Soria JC, Tan DSW, Chiari R, et al. First-line ceritinib versus platinum-based chemotherapy in advanced ALKrearranged non-small-cell lung cancer (ASCEND-4): a randomised, open-label, phase 3 study. Lancet 2017;389:917-29.

27. Camidge DR, Kim HR, Ahn MJ, et al. Brigatinib versus Crizotinib in ALK-Positive Non-Small-Cell Lung Cancer. N Engl J Med 2018;379:2027-39.

28. Welsh JW, Komaki R, Amini A, et al. Phase II trial of erlotinib plus concurrent whole-brain radiation therapy for patients with brain metastases from non-small-cell lung cancer. J Clin Oncol 2013;31:895-902.

29. Iuchi T, Shingyoji M, Sakaida T, et al. Phase II trial of gefitinib alone without radiation therapy for Japanese patients with brain metastases from EGFR-mutant lung adenocarcinoma. Lung Cancer 2013;82:282-7.

30. Mok TS, Wu YL, Ahn MJ, et al. Osimertinib or PlatinumPemetrexed in EGFR T790M-Positive Lung Cancer. N Engl J Med 2017;376:629-40.

31. Soria JC, Ohe Y, Vansteenkiste J, et al. Osimertinib in Untreated EGFR-Mutated Advanced Non-Small-Cell Lung Cancer. N Engl J Med 2018;378:113-25.

32. Sperduto PW, Kased N, Roberge D, et al. Summary report on the graded prognostic assessment: an accurate and facile diagnosis-specific tool to estimate survival for patients with brain metastases. J Clin Oncol 2012;30:419-25.

33. Gaspar LE, Scott C, Murray K, et al. Validation of the RTOG recursive partitioning analysis (RPA) classification for brain metastases. Int J Radiat Oncol Biol Phys 2000;47:1001-6.

34. El Rassy E, Botticella A, Kattan J, et al. Non-small cell lung cancer brain metastases and the immune system:

From brain metastases development to treatment. Cancer Treat Rev 2018;68:69-79.

35. Planchard D, Popat S, Kerr K, et al. Metastatic non-small cell lung cancer: ESMO Clinical Practice Guidelines for diagnosis, treatment and follow-up. Ann Oncol 2018;29:iv192-237.

36. Ali A, Goffin JR, Arnold A, et al. Survival of patients with non-small-cell lung cancer after a diagnosis of brain metastases. Curr Oncol 2013;20:e300-6.

37. Enders F, Geisenberger C, Jungk C, et al. Prognostic factors and long-term survival in surgically treated brain metastases from non-small cell lung cancer. Clin Neurol Neurosurg 2016;142:72-80.

38. Tang N, Guo J, Zhang Q, et al. Greater efficacy of chemotherapy plus bevacizumab compared to chemoand targeted therapy alone on non-small cell lung cancer patients with brain metastasis. Oncotarget 2016;7:3635-44.

39. Inal A, Kodaz H, Odabas H, et al. Prognostic factors of patients who received chemotherapy after cranial irradiation for non-small cell lung cancer with brain metastases: A retrospective analysis of multicenter study (Anatolian Society of Medical Oncology). J Cancer Res Ther 2018;14:578-82.

40. Gerdan L, Segedin B, Nagy V, et al. Brain metastasis from non-small cell lung cancer (NSCLC). Strahlenther Onkol 2014;190:64-7.

41. Sperduto PW, Yang TJ, Beal K, et al. Estimating Survival in Patients With Lung Cancer and Brain Metastases: An Update of the Graded Prognostic Assessment for Lung Cancer Using Molecular Markers (Lung-molGPA). JAMA Oncol 2017;3:827-31.

42. Morris PG, Reiner AS, Szenberg OR, et al. Leptomeningeal metastasis from non-small cell lung cancer: survival and the impact of whole brain radiotherapy. J Thorac Oncol 2012;7:382-5.

43. Remon J, Le Rhun E, Besse B. Leptomeningeal carcinomatosis in non-small cell lung cancer patients: A continuing challenge in the personalized treatment era. Cancer Treat Rev 2017;53:128-37.

Cite this article as: Muniz TP, de Jesus VH, Sousa VA, Barbosa MV, de Lima VC. A modified recursive partitioning analysis for predicting overall survival in patients with nonsmall cell lung cancer and central nervous system metastases. J Thorac Dis 2019;11(9):3909-3919. doi: 10.21037/jtd.2019.08.115 


\section{List of investigators}

(I) Thiago Pimentel Muniz, MD;

(II) Victor Hugo Fonseca de Jesus, MD;

(III) Victor Aurélio Ramos Souza, MD;

(IV) Malu Viter Rosa Barbosa, MD;

(V) Vladmir Cláudio Cordeiro de Lima, MD, PhD.

\section{Detailed inclusion and exclusion criteria}

\section{Inclusion criteria}

(I) Histologically or cytologically proven diagnosis of non-small cell lung cancer (NSCLC);

(II) Evidence (by imaging, pathological, or cytological analysis) of central nervous system (CNS) metastatic spread (parenchymatous and/or leptomeningeal), either at diagnosis or later in the course of disease;

(III) Age at diagnosis of CNS metastasis $\geq 18$ years;

(IV) Treatment of NSCLC initiated at A.C. Camargo Cancer Center;

(V) Patients who received any treatment, including best supportive care only.

\section{Exclusion criteria}

(I) Patients with any of the diagnosis below:

(i) Small cell lung cancer;

(ii) Large cell neuroendocrine carcinoma of the lung;

(iii) Typical or atypical neuroendocrine tumor of the lung;

(II) Patients with a secondary malignant neoplasia diagnosed either concomitantly or up to 5 years before the diagnosis of NSCLC;

(III) Patients who did not receive treatment for NSCLC at A. C. Camargo Cancer Center (e.g., patients who came for a second opinion);

(IV) Patients whose CNS lesions were considered to be related to another pathology (e.g., CNS infections, other malignant neoplasia);

(V) Patients with no more than two visits at A. C. Camargo Cancer Center;

(VI) Patients whose medical records were deemed insufficient.

\section{Definition of categories}

(I) Active smoker-a patient who was actively smoking tobacco at the time of diagnosis of NSCLC or who had stopped up to 3 months before the diagnosis of NSCLC.

(II) Former smoker-a patient who stopped smoking tobacco at least 3 months before the diagnosis of NSCLC.

(III) Synchronous diagnosis-the diagnosis of CNS metastasis occurred at the time of diagnosis of NSCLC or up to 3 months after the diagnosis of NSCLC. The diagnosis of a CNS lesion that ultimately led to the diagnosis of NSCLC was also considered synchronous.

(IV) Metachronous diagnosis-the diagnosis of CNS metastasis occurred at least 3 months after the diagnosis of NSCLC.

(V) Number of extracranial sites-lesions that occurred in an organ bilaterally (e.g., lungs, adrenals) were counted as one site. Bones were counted as one site, no matter how many bones were involved.

(VI) Systemic treatment only-whenever a patient received only systemic treatment as the first-line of therapy for the CNS metastasis (without association of a local treatment). If at intracranial progression a local treatment was administered, this was also considered as "systemic treatment only".

(VII) Concurrent systemic and local treatmentwhenever the local treatment was initiated on the first day of the first cycle of the systemic treatment or between the first and second cycle of the systemic treatment. If the systemic treatment was a tyrosine-kinase inhibitor, the local treatment was considered to be given concomitantly if administered within the first 30 days of the tyrosine-kinase inhibitor.

(VIII) Upfront systemic treatment-whenever a modality of systemic treatment was given alone as the first-line therapy for the CNS metastasis and a local treatment was associated after the second cycle of chemotherapy or immunotherapy, or after 30 days of initiation of a tyrosine-kinase inhibitor. 

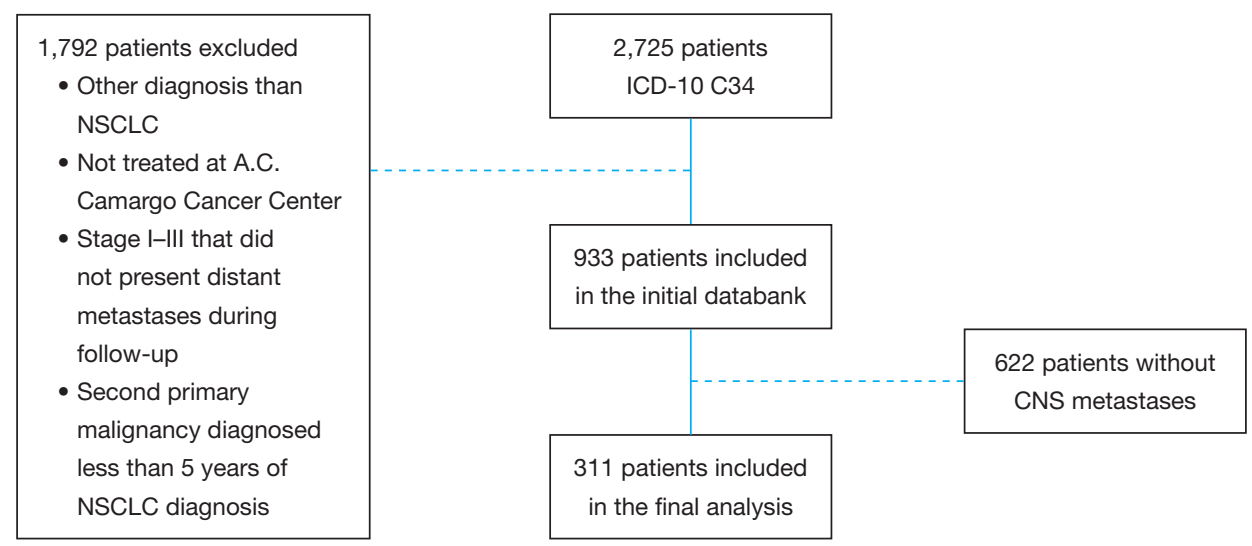

Figure S1 Patient selection flowchart. ICD-10, international classification of diseases 10th revision; CNS, central nervous system.
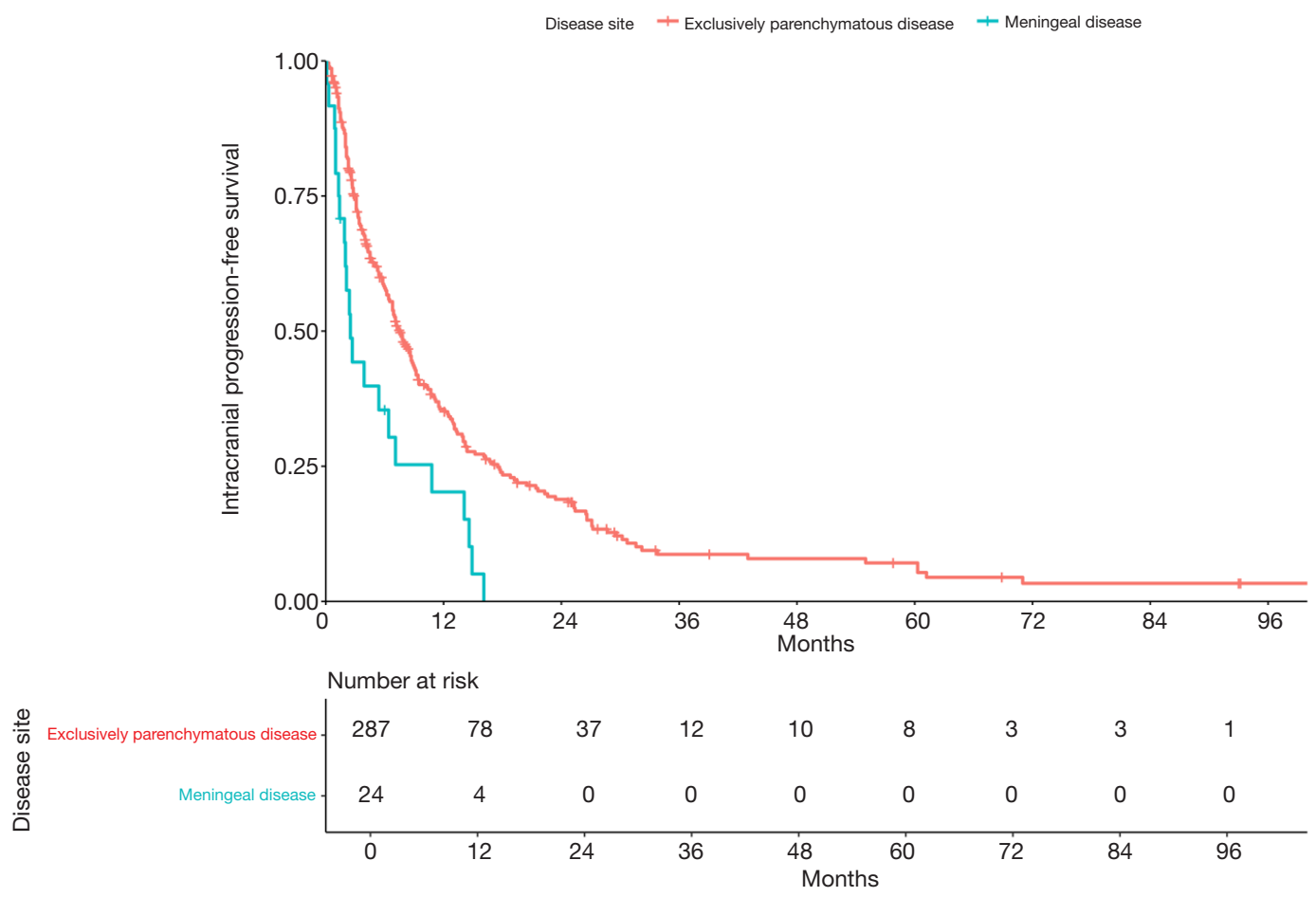

Figure S2 Intracranial progression-free survival in patients with exclusively parenchymatous disease vs. patients with leptomeningeal disease. Curves were calculated by the Kaplan-Meier method. 


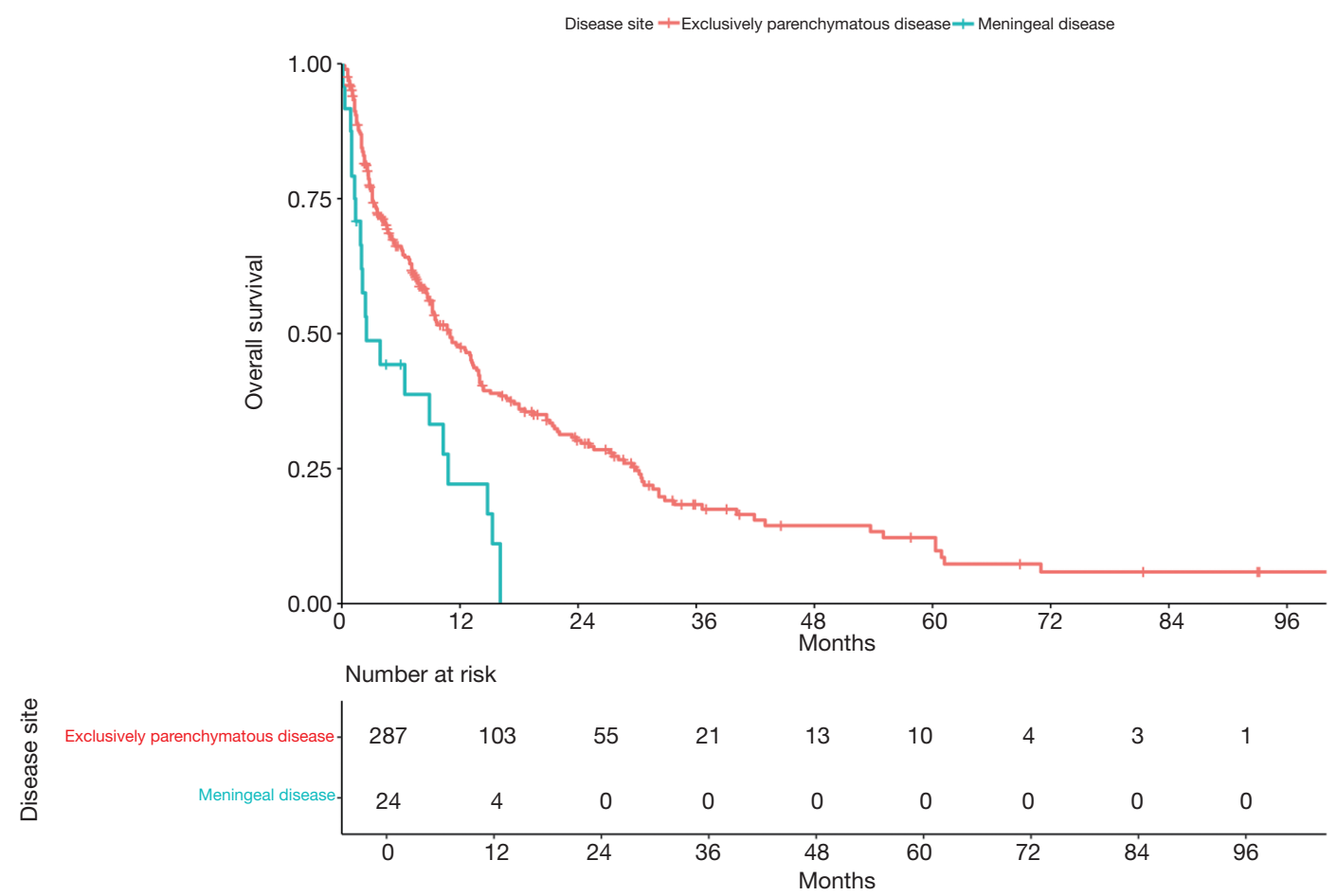

Figure S3 Overall survival in patients with exclusively parenchymatous disease vs. patients with leptomeningeal disease. Curves were calculated by the Kaplan-Meier method. 
Table S1 Univariate and multivariate analyses for intracranial progression-free survival ${ }^{\mathrm{a}}$

\begin{tabular}{|c|c|c|c|c|c|}
\hline Variable & $\mathrm{N}^{\mathrm{b}}$ & \multicolumn{2}{|c|}{ Univariate analysis } & \multicolumn{2}{|c|}{ Multivariate analysis $^{c}$} \\
\hline Age & 287 & $1.01(1-1.02)$ & 0.01 & $1.00(0.98-1.02)$ & 0.46 \\
\hline Charlson Comorbidity Index & 287 & $1.06(0.98-1.15)$ & 0.1 & $1.03(0.89-1.19)$ & 0.61 \\
\hline Largest diameter of largest lesion & 255 & $1.01(1-1.02)$ & 0.01 & $1.00(0.99-1.02)$ & 0.24 \\
\hline Active smoker & & 1 & & - & - \\
\hline Never smoker & & $0.89(0.63-1.26)$ & 0.52 & - & - \\
\hline Former smoker & & $0.85(0.61-1.19)$ & 0.36 & - & - \\
\hline ECOG performance status & 252 & & & & \\
\hline Histology & 274 & & & & \\
\hline Non-adenocarcinoma & & 1 & & - & - \\
\hline Adenocarcinoma & & $0.91(0.62-1.32)$ & 0.62 & - & - \\
\hline Known driver mutation $^{d}$ & 287 & & & & \\
\hline Yes & & 1 & & 1 & \\
\hline No & & $1.78(1.2-2.66)$ & $<0.01$ & $2.26(1.41-3.61)$ & $<0.01$ \\
\hline Time of diagnosis ${ }^{e}$ & 287 & & & & \\
\hline Synchronous & & 1 & & 1 & \\
\hline 4 or more & & $1.06(0.79-1.43)$ & 0.66 & $1.33(0.93-1.90)$ & $<0.01$ \\
\hline Number of extracranial sites ${ }^{e}$ & 287 & & & & \\
\hline 0 & & 1 & & 1 & \\
\hline 1 & & $1.32(0.88-1.97)$ & 0.17 & $1.30(0.77-2.18)$ & 0.31 \\
\hline 2 & & $1.57(1.04-2.36)$ & 0.03 & $1.42(0.85-2.37)$ & 0.17 \\
\hline 3 & & $1.15(0.71-1.87)$ & 0.55 & $0.96(0.51-1.82)$ & 0.92 \\
\hline 4 or more & & $1.89(1.15-3.11)$ & 0.01 & $1.43(0.78-2.61)$ & 0.24 \\
\hline
\end{tabular}

${ }^{\mathrm{a}}$, patients with meningeal carcinomatosis, and without complete data were excluded; ${ }^{\mathrm{b}}$, number of patients with complete data for each variable in univariate analysis; ' , two hundred and eighteen patients were included for multivariate analysis; ${ }^{\mathrm{d}}$, EGFR, ALK or ROS1; e, see supplementary appendix for definition of categories. HR, hazard ratio; Cl, confidence interval; ECOG, Eastern Cooperative Oncology Group; CNS, central nervous system. 
Table S2 Overall survival, according to modified recursive partitioning analysis (mRPA) classes ${ }^{\mathrm{a}}$

\begin{tabular}{|c|c|c|c|c|}
\hline Class & Prognostic factors & Overall survival (months) $(95 \% \mathrm{Cl})$ & $\mathrm{HR}(95 \% \mathrm{Cl})$ & $\mathrm{P}$ \\
\hline ॥ & ECOG $0-1$, no driver mutation ${ }^{\mathrm{b}} 1$ Brain Lesion & $18(12-29.5)$ & $2.01(1.01-3.99)$ & 0.04 \\
\hline III & ECOG $0-1$, no driver mutation ${ }^{b}>1$ brain lesion & $9.6(7.5-12.6)$ & 3.39 (1.79-6.43) & $<0.01$ \\
\hline IV & ECOG 2-4 & $3.1(2-7.1)$ & $6.08(3.06-12.10)$ & $<0.01$ \\
\hline
\end{tabular}

$\mathrm{HR}$, hazard ratio; $\mathrm{Cl}$, confidence interval; NR, not reached. ${ }^{a}$, patients with meningeal carcinomatosis, and without complete data were not included; ' , EGFR, ALK or ROS1.

Table S3 Overall survival, according to recursive partitioning analysis (RPA) classes ${ }^{\mathrm{a}}$

\begin{tabular}{lccccc}
\hline Class & $\mathrm{N}$ & Events & Overall survival (months) $(95 \% \mathrm{Cl})$ & $\mathrm{HR}(95 \% \mathrm{Cl})$ & 1 \\
\hline I & 9 & 6 & $16.4(3.1-\mathrm{NR})$ & $\mathrm{p}$ & $1.3(0.57-2.96)$ \\
II & 195 & 131 & $13(9.5-14.4)$ & $6.46(2.42-17.26)$ & 0.53 \\
III & 14 & 13 & $1.3(0.6-2.8)$ & $<0.01$ \\
\hline
\end{tabular}

a , patients with meningeal carcinomatosis, and without complete data were not included. $\mathrm{N}$, number of patients; HR, hazard ratio; Cl, confidence interval; NR, not reached.

Table S4 Overall survival, according to the graded prognostic assessment (GPA) score ${ }^{\mathrm{a}}$

\begin{tabular}{lccccc}
\hline GPA score & $\mathrm{N}$ & Events & Overall Survival (months) $(95 \% \mathrm{Cl})$ & $\mathrm{HR}(95 \% \mathrm{Cl})$ & 1 \\
\hline $0-1$ & 92 & 66 & $7.1(3.1-11.6)$ & $0.69(0.5-0.97)$ \\
$1.5-2.5$ & 110 & 75 & $13.3(9.7-17.1)$ & $0.36(0.13-1.01)$ & 0.03 \\
3 & 8 & 4 & $29.5(7.9-\mathrm{NR})$ & 0.053 \\
$>3$ & 8 & 5 & $19.5(6.9-\mathrm{NR})$ & $0.38(0.15-0.97)$ & 0.04 \\
\hline
\end{tabular}

a , patients with meningeal carcinomatosis, and without complete data were not included. N, number of patients; HR, hazard ratio; Cl, confidence interval; NR, not reached.

Table S5 Prognostic performance of mRPA, RPA, and GPA

\begin{tabular}{lccc}
\hline Statistic & mRPA & RPA & GPA \\
\hline AIC & $1,328.4$ & $1,344.1$ & 0.56 \\
C-statistic & 0.65 & 0.566 .1 & 0.59 \\
Bootstrap C-statistic (test dataset) & 0.65 & 0.58 \\
Bias-corrected Bootstrap C-statistic & 0.65 & 0.59 \\
\hline
\end{tabular}

mRPA, modified recursive partitioning analysis; RPA, recursive partitioning analysis; GPA, graded prognostic assessment; AIC, Akaike information criteria. 